\title{
HISTÓRIA, CULTURA MATERIAL E CONSTRUÇÕES IDENTITÁRIAS NA SENEGÂMBIA*
}

Ibrahima Thiaw ${ }^{* *}$

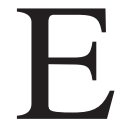

ste artigo utiliza dados arqueológicos e materiais e os confronta com as fontes clássicas da história para produzir uma ou mais histórias alternativas e esclarecer as trajetórias históricas sobre a gestão e a ocupação do espaço, as diferentes construções e reivindicações identitárias no espaço da Senegâmbia. Com esse objetivo, realizamos uma breve avaliação dos saberes e dos limites da produção arqueológica, histórica, antropológica e etnográfica dessa região.

As pesquisas em arqueologia histórica, ${ }^{1}$ em etnoarqueologia ${ }^{2}$ e

* Traduzido por Alba Valéria Tinoco da Silva e Marcelo Nascimento Bernardo da Cunha.

** Ibrahima Thiaw é Assistente de Pesquisa do Instituto Fundamental da África Negra (IFAN) da Universidade Cheikh Anta Diop de Dakar onde dirige o Laboratório de Arqueologia e o Museu Théodore Monod de Arte Africana do IFAN.

1 Susan Keech McIntoch e Ibrahima Thiaw, "Tools for Understandign Transformation and Continuity in Senegambian Society : 1500-1900", in C. R. Decorse (ed.), Est Africa During the Atlantic Slave Trade: Archaelogical Perspectives (New York : Lincester University Press, 2001), pp. 14-37; François G. Richard, "From Cosaan to Colony: Exploring Archaeological Landscapes Formations end Socio-Political Complexity in the Siin (Senegal), AD 500-1900" (Tese de Doutorado, Syracuse University, 2007); Ibrahima Thiaw, "The Gorée Archaeological Project (GAP): Material Things and the Use of Space in Plural Settings", Sephis Newsletter, n. 8 (2003) pp. 10-14.; Ibrahima Thiaw, "Archaeology and the Public in Senegal: Reflections on Doing Fieldwork at Home", Journal of African Archaeology, v. 1 (2) (2003) pp. 27-35; Ibrahima Thiaw, "The Gorée Archaeological Project (GAP): Preliminary Results", Nyame Akuma, n. 60 (2003) pp. 27-35; Ibrahima Thiaw, "The Built Environment and the Expansion of Social Dependence in Eighteenth Nineteenth Centuries in Land Senegambia (Upper Senegal River) ", Society of Historical Archaeology Newsletter, n. 31 (4) (1998), pp. 28; Ibrahima Thiaw, "An Archeological Investigation of Long-Term Culture Change in the Lower Falemme (Upper Senegal Region) AD 500-1900" (Tese de Doutorado, Rice University, 1999).

2 Agnès Gelbert, "Etude ethnoarchéologique des phénomènes d'emprunts céramiques. Enquêtes 
nas ciências sociais em geral têm demonstrado a dimensão material da vida social, oferecendo, assim, possibilidades de conexão entre a cultura material e as questões de memória e identidade. ${ }^{3}$

$\mathrm{Na}$ Senegâmbia, a arqueologia, até o momento, tem exercido um papel pouco reconhecido nesse domínio. A rigidez das fronteiras disciplinares fez com que ela se restringisse ao estudo dos períodos ditos "pré-históricos" ou "proto-históricos", que, fora dos círculos acadêmicos, não são geralmente conhecidos pelas populações locais e pelos griots ou outros comunicadores tradicionais. A arqueologia tinha então por missão documentar os períodos que a tradição oral e as fontes escritas não podiam esclarecer. ${ }^{4}$

Começaremos por "problematizar" as noções de fronteiras étnicas ou culturais e as influências externas. Buscaremos saber se a suposta homogeneidade étnica das regiões culturais e étnicas bem como as reivindicações e as construções identitárias que lhe são associadas têm assinaturas particulares sobre as culturas materiais e a organização do espaço.

Geralmente, antropólogos e historiadores supõem que se pode conhecer a "África tradicional" simplesmente descartando as alterações causadas pela imposição do governo colonial. ${ }^{5}$ Supõe-se, assim, que o período de mudanças globais a partir do último milênio é escopo exclusivo de historiadores e antropólogos. Mas a escrita, que é sua principal fonte de informação, bem como a oralidade, reflete e privilegia as ambições daqueles que as dominam. Assim, nossa história oficial é aquela dos privilegiados, dos reis e de suas cortes, dos estados e os dos homens

dans les Haute et Moyenne Vallées du Fleuve Sénégal (Sénégal)" (Tese de Doutorado, Université Paris X, 2000); N.S. Guèye, "Poteries et peuplements de la Moyenne Vallée du Fleuve Sénégal du XVI ${ }^{e}$ au XIX ${ }^{\mathrm{e}}$ siècle: approches ethnoarchéologique et ethno-historique" (Tese de Doutorado, Université de Paris X, 1998); Moustapha Sall, "Traditions céramiques, Identités et peuplement en Sénégambie. Ethnographie comparée et essai de reconstitution historique" (Tese de Doutorado, Université Libre de Bruxelles, 2001) ; Manidiomé Thiam, "La céramique au Sénégal: archéologie et histoire" (Tese de Doutorado, Université de Paris I, 1991).

Arjun Appadurai. (ed.), The Social Life of Things: Commodities in Cultural Perspective, Cambridge: Cambridge University Press, 1986; Michel-Rolph Trouillot, Silencing the Past. Power and the Production of History, Boston: Beacon Press, 1995.

4 Thiaw, "The Gorée Archaeological Project (GAP): Preliminary Results", pp. 27-35.

5 Thiaw, "An Archeological Investigation of Long-Term Culture Change in the Lower Falemme" 
de estado. De súbito, cai-se nas armadilhas e nos estereótipos da "biblioteca colonial". 6

\section{Espaços e identidades}

Do ponto de vista das identidades, a Senegâmbia é um mosaico de espaços e populações, cujas histórias foram configuradas em decorrência de conflitos, negociações e acordos ao longo dos anos. A despeito dos esforços dos poderes coloniais e pós-coloniais com suas cartografias e suas múltiplas tecnologias de controle, visando fazer coincidir identidade e território, essa região é culturalmente um conjunto plural, um caleidoscópio de nações étnicas, correspondendo a identidades mais ou mesmo distintas. Em face de tal diversidade, o abade Boilat, ${ }^{7}$ um padre francosenegalês do século XIX, surpreende-se que populações, vivendo em um mesmo ambiente, tendo acesso aos mesmos tipos de recursos e submetidas aos mesmos riscos e doenças, pudessem exibir tantas diferenças no âmbito do físico, do linguístico e da organização sociopolítica. ${ }^{8}$

O modelo islamo-wolof e seu projeto de wolofização como fator de homogeneização da sociedade senegalesa moderna é geralmente considerado como um marco que conduzirá ao desaparecimento das identidades não wolof. ${ }^{9}$ No Senegal, essa tensão entre o estado supranacional, relacionado a um imaginário da modernidade, e as construções identitárias, associadas a um imaginário da tradição, é exacerbada por uma outra contradição: aquela opondo os wolof a seus vizinhos. Na realidade, o estado pós-colonial parece fundar-se sobre um modelo islamowolof, inspirado no poder colonial que relegou as outras etnias à periferia. O que leva à questão da wolofização.

6 Ann B. Stahl, Making History in Banda. Anthropological Visions of Africa's Past, Cambridge: Cambridge University Press, 2001.

7 Abbé Pierre David Boilat, Esquisses sénégalaises, Paris: Karthala, 1984.

8 Thiaw, "Archaeology and the Public in Senegal", p. 222

9 Momar Coumba Diop e Mamadou Diouf, Le Sénégal sous Abdou Diouf: Etat et Société, Paris: Karthala, 1990; Makhtar Diouf, Sénégal: les ethnies et la nation, Dakar: Nouvelles Editions Africaines du Sénégal, 1998; Donal Cruise O’Brien, "Langue et nationalité au Sénégal: l'enjeu politique de la wolofisation", in M.-C. Diop et M. Diouf (eds), La construction de l'État au Sénégal (Paris: Karthala, 2002), pp. 143-55. 
Esta wolofização provém daquilo que Donald Cruise O"Brien ${ }^{10}$ chama de acidentes históricos: as confrarias (a wolofização pelo sagrado), as quatro divisões administrativas, ${ }^{11}$ a cultura do amendoim, a escola, a estrada de ferro e a administração. A tais fatores geradores podem-se agregar atualmente os fatores da evolução, como os meios de massa, principalmente audiovisuais, que transmitem geralmente em língua wolof. É necessário também acrescentar o êxodo rural, sobretudo depois das grandes secas da década de setenta, e seu consequente desenvolvimento urbano, no qual o wolof funciona como língua franca.

A definição de identidade surge de uma dupla construção. Há uma valorizadora de si (isso que Matar Diouf chama de endopercepção), ou seja, o modo como os membros da etnia se percebem, ${ }^{12}$ que, ao mesmo tempo, faz apelo a uma segunda que frequentemente exclui e desvaloriza o outro (isso que Matar Diouf chama de exopercepção), ou seja, o modo como os membros identitários são percebidos, sem deixar claro como estes percebem os outros. Entre os wolof, trata-se da noção de lakkat que evidencia essa dupla construção. De um lado, há os wolof que se percebem como um grupo civilizado e, do outro, os lakkat, ou seja, os não wolof que algaraviam e falam uma língua ininteligível. Além disso, Cruise O'Brien ${ }^{13}$ os define como "uma pessoa que emite sons estranhos e ininteligíveis".

De todo caso, a preponderância de língua wolof e a reterritorialização da cultura islâmica exerceram um papel determinante no processo de wolofização. Assim, a sociedade senegalesa parece dividir-se entre dois grandes grupos: os wolof e os lakkat. Segundo Matar Diouf, ${ }^{14} \mathrm{o}$ lakkat significa literalmente "aquele que fala uma língua". Aqui, tratase pura e simplesmente do africano, cuja língua materna não é o wolof: o que inclui os senegaleses não wolof, mais particularmente aqueles do grupo linguístico Mande, bem como todos os outros africanos.

\footnotetext{
10 O’Brien, “Langue et nationalité au Sénégal”, pp. 143-55.

11 As quatro cidades sede da administração colonial francesa situadas no Senegal: Dakar, Goreé, Rufisque e Sant-Louis.

12 Diouf, Sénégal: Les ethnies et la nation, p.10.

13 O’Brien, "Langue et nationalité au Sénégal", p. 150.

14 Diouf, Sénégal: Les ethnies et la nation, p. 47
} 
Convém notar que os teóricos da wolofização privilegiam a língua e a religião em detrimento de outros critérios de definição étnica ou identitária, que se podem destacar dos habitus ${ }^{15}$ que deixam rastros materiais ou que são intangíveis, mas que nem por isso deixam de ser formas de expressão tão valiosas quanto a língua. Esses habitus são os comportamentos, os gestos e as ações rotineiras, relacionadas às práticas da vida cotidiana e que se materializam ou não no espaço, mas que possuem uma profunda ancoragem no patrimônio cultural de uma dada comunidade. Assim como a língua, estão sujeitos à mudança, embora também possam perdurar na longa duração e surjam como modos de contestação e de negociação identitária, sobretudo dentro do esquema de uma sociedade estatal. ${ }^{16}$

Assim como a língua, a cultura material e as formas intangíveis da identidade são também poderosos vetores de transmissão, de afirmação e de manutenção da identidade. As pesquisas etnoarqueológicas no espaço senegambiano colocam em evidência a persistência de assinaturas etnoculturais específicas. ${ }^{17}$ Há certos elementos de convergência e interação, mas as marcas da hegemonia wolof são menos aparentes. Outros elementos são oriundos, sobretudo, da modernidade global, como o plástico ou outros objetos importados, que constituem a ameaça mais visível para essas expressões identitárias materiais.

Além disso, diante da questão "a que grupo étnico você pertence?", numerosos locutores wolofizados respondem: "meu pai, ou minha mãe, ou meu avô ou minha avó era sereer, sosse, joola, manding, pël, tukulör etc.". Ao que me parece, não se trata de uma reivindicação de identidade étnica wolof ou daquela dos pais, avós e bisavós. Daí a adoção de uma nova língua ou de uma nova religião pode ser o produto de uma mudança identitária mais superficial do que parece e é falacioso

15 Pierre Bourdieu, Esquisse d'une théorie de la pratique : précédée de trois études d'ethnologie kabyle, Genève : Editions Droz, 1972

16 Ana Maria Alonso, "The Politics of Space, Time and Substance : State Formation, Nationalism and Ethnicity", Annual Review of Anthropology, 23 (1994), pp. 379-405.

17 Gelbert, "Etude ethnoarchéologique des phénomènes d'emprunts céramiques"; Guèye, "Poteries et peuplements de la Moyenne Vallée du Fleuve Sénégal"; Sall, "Traditions céramiques, identités et peuplement en Sénégambie".; Thiam, "La céramique au Sénégal". 
pensar que a nova identidade que daí emergirá será equivalente àquela do grupo hegemônico ou dominante.

As cartografias das identidades e das etnias na Senegâmbia parecem sugerir espaços de povoamento historicamente homogêneos. ${ }^{18} \mathrm{~A}$ divisão da população, na época histórica, foi utilizada como modelo para compreender a distribuição étnica dos períodos mais antigos, particularmente na Idade do Ferro. Assim, o Fuuta é geralmente considerado como o epicentro da cultura halpulaar; o noroeste da Senegâmbia, o país wolof; o Siin Salum, o país sereer; a região ao sul da República da Gâmbia, o país diola; a região leste, aquela das minorias soninké e bambara, bassari, bedik etc.

Esa cartografia esquemática oculta, em muitos aspectos, a complexidade e a dinâmica histórica do povoamento. ${ }^{19}$ Ainda que os dados linguísticos pareçam apoiar tal subdivisão, é difícil que, desta, aqueles possam definir os contornos, os processos, o grau e a interação dos diferentes grupos na escala local, o papel e a amplitude do contato com grupos estrangeiros na escala regional, inter-regional e internacional.

\section{Vestígios arqueológicos e identidades}

A periodização do passado da África e da Senegâmbia, em paleolítico, neolítico, proto-história ou Idade do Ferro, história, foi objeto de muitos debates aos quais nos parece inútil retornar. Esclarecemos que a utilização desses termos no esquema de nossa pesquisa é puramente convencional. Durante o período colonial, o centro da pesquisa arqueológica na Senegâmbia foi, sobretudo, a pré-história (o paleolítico e o neolítico), antes de ser, atualmente, relegada ao segundo plano. A crescente falta de interesse pela pré-história pode estar ligada à ausência de tradições e reivindicações dos sítios pertencentes a esse período.

Da independência, em 1960, até os anos 90, a Idade do Ferro foi o objeto de maior atenção. Ela abrange o período que vai do início do primeiro milênio de nossa era até o século XV. Constitui uma fase crucial

18 Boilat, Esquisses sénégalaises; Diouf, Sénégal: les ethnies et la nation.

19 Diouf, Sénégal: Les ethnies et la nation. 
na formação das elites, na estratificação social, no desenvolvimento de um comércio a longa distância, na expansão do Islã etc.

De uma perspectiva da longa duração, seria possível conceber vários tipos de espaços contribuindo para estruturar as sociedades africanas: de trocas, estatais, linguísticos, culturais e religiosos. ${ }^{20}$ Também as inovações, sejam elas produto de fatores e de forças internas ou externas ligadas ao contato com os grupos estrangeiros, afetaram em graus variáveis esses diferentes espaços. A expressão mais evidente dessas diferenças na cultura material é perceptível no que se convencionou chamar, atualmente, de as quatro províncias proto-históricas da Senegâmbia, que coincidiram com a Idade do Ouro ou do Senegal. ${ }^{21}$ Essas principais províncias culturais são: os sítios do Vale do Rio Senegal, os túmulos de areia, os sambaquis e os megálitos.

Trabalhos mais recentes mostram que a diferenciação da cultura material sobre a qual se apoia essa distinção era de natureza cronológica no Vale do Rio, ${ }^{22}$ e é bem provável que esse seja também o caso dos sambaquis. $^{23}$

A subdivisão de Idade do Ferro da Senegâmbia em províncias ou áreas culturais é mais problemática. Ela reparte os vestígios a partir de uma base material e identitária. Com exceção do Vale do Rio, os espaços culturais definidos o são a partir de monumentos e de rituais funerários (túmulos, sambaquis e megálitos), e dão o aspecto de reinos onde as populações não viveram senão nas suas tumbas.

20 Jean Pierre Chrétien e Gerárd Prunier (eds.), Les ethnies ont une histoire, Paris: Karthala, 2003; Jean-Luc Amselle e Elikia M'Bokolo (eds.), Au coeur de l'ethnie: ethnie, tribalisme et Etat en Afrique Paris: Editions La Découverte \& Syros, 1999.

${ }^{21}$ Musée de Solutré, L’Age d'Or du Sénégal. Musée départemental de Préhistoire de Solutré, 1993.

22 Susan Keech McIntosh et al., "The Middle Senegal Valley Project: Preliminary Results from the 1990-91 Field Season", Nyame Akuma n. 38 (1992) pp. 47-61; Susan Keech McIntosh e H. Bocoum, "New Perspectives on Sincu Bara, a First Millennium Site in the Senegal Valley", African Archaeological Review, v.17, n. 1 (2000), p.1-43; Susan Keech McIntosh e H. Bocoum, Excavations at Sincu Bara, Middle Senegal Valley (Senegal), Nouakchott-Dakar: CRIAAIFAN, 2002.

23 Ibrahima Thiaw, "Développement touristique et mal gestion des ressources culturelles archéologiques dans le Delta du Saloum (Sénégal)". Comunicação no colóquio "Les perspectives de l'archéologie préventive en Afrique de l'Ouest", Nouakchott, IMRS-INRAP, 1-3 fevereiro de 2007. 
Considerada como o período de formação das diferentes identidades étnicas e das entidades políticas pré-coloniais, a Idade do Ferro ocupa um lugar de destaque nas reconstruções histórico-acadêmicas do espaço senegambiano. ${ }^{24}$ No contexto das lutas pela independência e pela afirmação de uma identidade nacional, esse período é um poderoso marco identitário para a nova elite intelectual local, consciente das manipulações e das tentativas de apagamento de seu passado pelo poder colonial e, mais tarde, pelo poder pós-colonial, que estão associados ao modelo islamo-wolof, descrito mais acima. O poder colonial em particular negava toda a historicidade e toda a criatividade cultural aos grupos locais, chegando até a se apropriar de certos monumentos percebidos como oriundos da difusão, a partir de outros focos de civilizações pré-determinadas. ${ }^{25}$ É nessa perspectiva que é necessário compreender toda a obra intelectual de Cheikh Anta Diop, ${ }^{26}$ buscando contrapor-se a essa abordagem colonial. Para Cheikh Anta Diop, ${ }^{27}$ a apropriação do passado africano pelos Africanos era a única via possível para por fim à arrogância do poder colonial.

Após a sequência nacionalista dos três primeiros decênios de independência, o novo interesse pela etnoarqueologia e pelos períodos históricos recentes é uma mudança paradigmática, que busca responder muito mais à demanda de história das populações locais do que a um imaginário nacionalista, inspirado pelo modelo imperial europeu. De fato, os sítios históricos recentes são objeto de reivindicações e de contestações intensas em relação às identidades. Em contrapartida, com exceção dos sítios do Vale do Rio Senegal, atribuídos aos Sereer pelas tradições, ${ }^{28}$ esses

${ }^{24}$ Hamady Bocoum, "La métallurgie du fer au Sénégal. Approches archéologique, technologique et historique" (Tese de Doutorado de $3^{\mathrm{e}}$ ciclo, Université de Paris I, 1986); Hamady Bocoum, L'Âge du Fer au Sénégal: histoire et archéologie. Nouakchott : IFAN-UCAD et CRIAA, 2000; Chavane, Villages de l'ancien Tekrour; Guy Thilmans e Annie Ravisé, Protohistoire du Sénégal. Recherches archéologiques, Tomo 2, Sinthiou Bara et les sites du Fleuve, Dakar: Mémoires de l'Institut Fondamental d'Afrique Noire, 1980.

25 Dr. Jouenne, "Les monuments mégalithiques du Sénégal, les roches gravées et leur interprétation culturelle", Bulletin du Comité d'Etude Historique et Scientifique de L'A.O.F. , v. 13, n. 3 (1930) pp. 309-99.

26 Cheikh Anta Diop, Nations Nègres et Cultures, Paris: Présence Africaine, 1979.

27 Diop, Nations Nègres et Cultures.

28 V. Martin e C. Becker, Répertoire des sites protohistoriques du Sénégal et de la Gambie, Kaolack: Ronéotypé, 1974; V. Martin e C. Becker, Inventaire des sites protohistoriques de Sénégambie. Kaolack: Ronéotypé, 1984; Chavane, Villages de l'ancien Tekrour. 
últimos guardam uma lembrança muito vaga sobre os autores da maior parte dos vestígios da Idade do Ferro. Como os sítios paleolíticos e neolíticos, aqueles da Idade do Ferro são raramente reivindicados, uma vez que são percebidos como obra de populações pré-islâmicas. ${ }^{29} \mathrm{~A}$ expansão do Islã, ao longo da segunda metade do segundo milênio, foi acompanhada por mudanças identitárias e pela negação das heranças pré-islâmicas pela grande massa de convertidos, sobretudo com o desenvolvimento do ativismo islâmico a partir do século XIX.

Isso foi produto da falta de profundidade da tradição oral ou o resultado de um longo processo de silenciamento e de apagamento de uma história conturbada? Essa questão nos parece pertinente, pois certos sítios datam da primeira metade do segundo milênio de nossa era talvez mesmo mais tardiamente - estando, portanto, ao alcance da tradição oral.

A falta de interesse das populações pelos sítios antigos (Idade do Ferro e pré-história) poderia ser também resultante dos esforços do regime colonial, que buscou apropriar-se dos elementos que o valorizassem e a apresentar os outros como primitivos, para demosntrar a ausência de historicidade das sociedades africanas. Essa política foi continuada pelo regime pós-colonial, que favorece, ainda hoje, os vestígios do passado colonial. As construções e os edifícios coloniais aparecem em destaque na lista de locais, classificados do patrimônio nacional do Senegal. O estado pós-colonial, contudo, esforçou-se para incorporar a essa lista os espaços dos "heróis nacionais" na resistência à colonização, promovendo assim os colonos e as figuras locais da resistência. ${ }^{30}$

As identidades das populações senegambianas não ficaram inertes desde a Idade do Ferro, permanecendo em mudança constante, graças a fatores históricos, políticos e socioeconômicos. De maneira que a expansão do Islã, a partir do século XI, terá por corolário uma reconfiguração das identidades, sobretudo na metade norte da Senegâmbia. É necessário acrescentar aí os movimentos das populações,

29 Thiaw, "Archaeology and the Public in Senegal", pp 27-35.

30 Thiaw, "The Gorée Archaeological Project (GAP): Preliminary Results", pp. 27-35.; Ibrahima Thioub, "L'école de Dakar et la production d'une écriture académique de l'histoire", in Diop M.C. (ed.), Le Sénégal contemporain (Paris: Karthala, 2002), pp. 109-53. 
decorrentes da degradação progressiva das condições climáticas. ${ }^{31} \mathrm{~A}$ expansão territorial dos Mande, a partir do século XIII, e, mais tarde, a do Jolof, que será portadora da cultura wolof, afetarão profundamente a composição étnica e as estruturas sociais de vários grupos no espaço senegambiano. O surgimento de novas identidades, como a dinastia Guelwaar que reinará no Siin, a partir do século XIV, por exemplo, contribui para redesenhar a cartografia étnica dessa região. ${ }^{32}$

Ao mesmo tempo, nota-se uma propagação de grupos socioprofissionais endogâmicos, comumente chamados "castas" na literatura, que aparecem como categorias identitárias supraétnicas. ${ }^{33}$ Alguns desses grupos, pelo monopólio que exercem sobre certas atividades, tais como o trabalho de cerâmica ou de metalurgia do ferro, podem ter uma grande visibilidade nos depósitos arqueológicos. Outros como os griots, cujas culturas materiais relacionadas são perecíveis, são menos visíveis na longa duração, mas se mantêm, porque são depositários da memória coletiva e aparecem como os mestres incontestáveis da produção e da reprodução das tradições orais.

A divisão sexual do trabalho é também um elemento determinante, pois certas especializações são apanágio exclusivo das mulheres e outras, dos homens. De modo que, nas sociedades hierarquizadas, por exemplo, o trabalho da cerâmica está ou nas mãos das mulheres dos griots, ou entre aquelas dos ferreiros ou dos tecelões. Tal não parece ser o caso nas sociedades descentralizadas e segmentadas, como entre os diola ou os grupos saafi, em que esta atividade permanece o apanágio das mulheres, sem que elas sejam necessariamente consideradas como "gente de casta".

A distribuição da cultura material posterior ao século XV também deixa transparecer grandes mudanças. Os conjuntos recentes atribuídos a esse período mostram uma relativa homogeneidade de formas

31 Alioume Déme, "Evolution climatique et processus de mise en place du peuplement dans l'Ile à Morphil" (Dissertação de Mestrado, Université Cheikh Anta Diop, 1991).

32 Henry Gravrand, La civilisation sereer. Cosaan: les origines, Dakar: Nouvelles Editions Africaines, 1983; Henry Gravrand, La civilisation Sereer. Pangool. Le génie religieux sereer, Dakar: Nouvelles Editions Africaines du Sénégal, 1990.

33 David C. Conrad e Barbara E. Frank (eds), Status and Identity in West Africa, Bloomington: Indiana University Press, 1995; Tal Tamari, "The Development of Caste System in West Africa", $J A H$, n.32 (1991) pp. 221-50. 
e decorações cerâmicas na metade norte da Senegâmbia e na região de Falemme. ${ }^{34}$ Tratam-se, geralmente, de produtos de troca especialmente de contas de vidro, mas também de cachimbos de manufatura local, em sua maioria.

As mudanças intervenientes ao longo do fim do primeiro e do segundo milênio foram documentadas principalmente pelos estudos sobre a cultura material cerâmica. Entretanto, nem todas as operações técnicas da cadeia operatória da cerâmica têm a mesma estabilidade, e sua variabilidade depende, em parte, de sua visibilidade, sua flexibilidade ou da relação com o contexto social. As técnicas que deixam traços visíveis sobre o produto acabado, principalmente a decoração, a seleção, a extração e o cozimento, são caracterizadas por certa visibilidade e uma maleabilidade técnica no tempo e no espaço. Essas etapas podem ser influenciadas por outros múltiplos fatores e, em consequência, não refletem mais do que facetas superficiais, situacionais e temporárias de identidade.

Em contrapartida, a técnica de modelagem inicial, para falar propriamente, não deixa traços visíveis sobre o produto acabado e depende dos gestos especializados ou habitus adquiridos no curso da aprendizagem, e que podem ser bastante estáveis durante um longo período em um dado espaço. É nesse nível de análise que se têm maiores chances de encontrar os aspectos mais marcantes da identidade, particularmente o parentesco, a linguagem, o gênero e a classe.

Na Senegâmbia, a maior parte dos estudos de cerâmica põe em destaque as decorações e as formas que têm geralmente uma ampla distribuição no espaço, que não parecem coincidir com as categorias identitárias específicas. Os trabalhos em etnoarqueologia sobre a produção cerâmica de Guèye, ${ }^{35}$ Sall $^{36}$ e Thiam ${ }^{37}$ preenchem esse vazio e autori-

${ }^{34}$ Guèye, "Poteries et peuplements de la Moyenne Vallée du Fleuve Sénégal"; Susan Keech McIntosh e Roderick J. McIntosh, "Field Survey in the Tumulus Zone in Senegal", The African Archaeological Review, n.11 (1993) pp. 73-107; Thiaw, "An Archeological Investigation of Long-Term Culture Change in the Lower Falemme"; Thilmans e Ravisé, Protohistoire du Sénégal.

35 Guèye, "Poteries et peuplements de la Moyenne Vallée du Fleuve Sénégal"

${ }^{36}$ Moustapha Sall, Traditions céramiques, identités et peuplement en Sénégambie. Ethnographie comparée et essai de reconstitution historique, British Archaeological Reports, 2005.

37 Thiam, "La céramique au Sénégal" 
zam correlações possíveis entre identidades e cultura material, particularmente entre os Halpulaar do Vale do Rio, os Sereer do Siin-Saloum, o país Fogny em Casamance, os Bassari e os Bedik do Senegal Oriental.

A aceitação, às vezes ingênua, das tradições orais sem a interpretação dos vestígios arqueológicos deveria levar em conta as considerações ideológicas e as relações de força, passadas e presentes, que ligam as populações que convivem nessas regiões. ${ }^{38}$ Nota-se também, na literatura histórica, uma confiança quase absoluta no que tange aos dados arqueológicos que são tomados como provas irrefutáveis.

É certo que a arqueologia pode trazer sua contribuição para esse debate, mas é forçoso reconhecer que se não for dentro de um contexto etnográfico ou etnoarqueológico, a atribuição de uma cultura material a uma identidade específica é um verdadeiro quebra-cabeça - na arqueologia - e requer uma metodologia rigorosa, muitas vezes ausente na maior parte dos trabalhos centrados sobre essa tarefa no espaço senegambiano.

Os cinco últimos séculos foram marcados pelo desenvolvimento da economia atlântica, o tráfico de escravos e uma grande mobilidade de bens materiais e de pessoas, que foram acompanhadas de uma mistura de identidades adquiridas durante a Idade do Ferro e de uma recomposição das fronteiras e das categorias identitárias. A expansão do sistema capitalista foi seguida, a partir da segunda metade do século XIX, por uma imposição de novas lógicas de distinções identitárias pelo governo imperial europeu.

Em face dessa nova dinâmica, as diferentes populações senegambianas elaboraram estratégias e respostas com base em suas respectivas experiências históricas. A compreensão do impacto desses processos sobre as populações locais passa, então, necessariamente, por se levarem em conta as especificidades na escala local em que se elaboram as respostas dos diferentes grupos e categorias identitárias.

Esee período foi largamente investigado pelos historiadores em razão da existência de documentos escritos e tradições orais ainda vigo-

${ }^{38}$ Y.K. Fall, "Silla: Problématique d'un site de la vallée du fleuve", Archives suisses d’Anthropologie générale, Genève, n. 46 (1982) p.199-216. 
rosas. ${ }^{39}$ Uma análise rigorosa das fontes com base na linguagem (documentos escritos e orais) permitiu a esses autores apresentar um quadro coerente das transformações nas sociedades senegambianas, ao longo dos cinco últimos séculos.

Entretanto, eles dão destaque, sobretudo, aos europeus, às aristocracias locais, aos sacerdotes e aos comerciantes, considerados como os "produtores de história por excelência", porque controlam a seleção, o arquivamento, a produção e a transmissão das informações históricas. De maneira que, na maioria dos casos, os indivíduos comuns, marginalizados, mas, de resto, bastante implicados no processo em curso, têm uma baixa visibilidade nessas construções históricas.

A arqueologia histórica, embora em seu início, fornece informações sobre os diversos atores, através dos estudos dos sítios e das culturas materiais. ${ }^{40}$ Por conseguinte, pode contribuir para os debates relacionados à incorporação das sociedades senegambianas nas correntes de mudanças do sistema capitalista e no seu impacto sobre a vida cotidiana. Está mesmo em uma posição privilegiada para avaliar a importância, a natureza e o papel dos produtos das trocas nas economias locais, em razão do caráter fragmentário das fontes escritas a esse respeito.

39 Boubacar Barry, Le royaume du Waalo. Le Sénégal avant la conquête, Paris: Karthala, 1985; Boubacar Barry, La Sénégambie du XVe au XIXe siècle: traite négrière, Islam et conquête coloniale, Paris: L’Harmattan, 1988; Abdoulaye Bathily, Les Portes de l'or. Le Royaume de Galam (Sénégal) de l'ère des musulmans au temps des négriers (VIIIe-XVIIIe siècle), Paris: L'Harmattan, 1989; Jean Boulègue, Les anciens royaumes wolof (Sénégal): Le grand Jolof (XIIIe-XVIe siècle), Paris: Editions Façades, 1987. ; Andrew F. Clark, From Frontier to Backwater: Economy and Society in the Upper; Senegal Valley (West Africa), 1850-1920, Lanham: University Press of America, 1999 ; Philip. D. Curtin, Economic Change in Precolonial Africa. Senegambia in the Era of the Slave Trade, Madison: The University of Wisconsin Press, 1975; Mamadou Diouf, Le Kajoor au XIXe siècle: Pouvoir ceddo et conquête coloniale, Paris: Karthala, 1990; Rokhaya Fall, "Le royaume du Baol du XVIè au XIXè siècle. Pouvoir wolof et rapports avec les populations sereer" (Tese de Doutorado de $3^{\mathrm{e}}$ ciclo de história, Université de Paris I, 1983) ; Michael. A. Gomez, Pragmatism in the Age of Jihad. The Precolonial State of Bundu, Cambridge: Cambridge University Press, 1992; Martin Klein, Islam and Imperialism in Senegal: The Sine-Saloum, 1847-1914, Stanford: Stanford University Press, 1968; Francois Manchuelle, Willing migrants. Soninke labor diasporas, 1848-1960, Athens: Ohio University Press, 1997; David Robinson, The Holy War of Umar Tal: The Western Sudan in the Mid-Nineteen Century, Oxford: Clarenton Press, 1985; James F. Searing, West African Slavery and Atlantic Commerce. The Senegal River Valley, 1700-1860, Cambridge: Cambridge University Press, 1993.

40 Richard, "From cosaan to colony"; Thiaw, "An archeological investigation of long-term culture change in the Lower Falemme", 27-35 


\section{Conclusão}

Situada nos limites meridionais do Saara e nas portas do Atlântico, a Senegâmbia atraiu o olhar dos negociantes e dos cronistas árabes, desde o início do segundo milênio da nossa era, em seguida dos exploradores europeus, a partir do século XV. Dispõe, dessa forma, de um rico corpus historiográfico para a reconstrução das trajetórias históricas de diferentes experiências culturais que ali são expressas.

Tais vantagens aparentes escondem, entretanto, uma série de armadilhas, que se refletem nas reescrituras da história na era pós-colonial. O impacto e as consequências da incorporação dessa região à economia mundial dos sistemas trans-saariano e atlântico, por exemplo, permanecem particularmente controversos, em razão - em parte - do caráter estereotipado, incompleto e tendencioso da biblioteca histórica, cuja produção coincide com o início da marginalização da África na história universal.

Na Senegâmbia, como em qualquer parte do mundo, a história é intensamente negociada no presente. ${ }^{41}$ Como tal, a produção histórica está também intrinsecamente ligada às relações de forçaa ${ }^{42} \mathrm{O}$ mesmo se dá com as construções e as reivindicações identitárias, que são fundamentalmente imaginadas.

A cultura material é suscetível de fornecer informações-chave sobre a história social e a questão das identidades, mas isso requer uma metodologia de análise rigorosa. Em última análise, essas informações devem ser confrontadas às fontes documentais e orais. Essa manobra uma vez que lança um olhar crítico sobre a biblioteca colonial e póscolonial, sobre a história oficial, as reivindicações identitárias e as fronteiras entre os grupos e os espaços de uma determinada região - permite renovar o conhecimento da dinâmica humana e das relações culturais.

Estima-se que a arqueologia possa favorecer um conhecimento mais inclusivo, pois a produção, o consumo, a rejeição e a reciclagem

${ }^{41}$ David William Cohen e E.S.Atieno Odhiambo, Siaya: The Historical Anthropology of an African Landscape, London: James Currey, 1989.

${ }^{42}$ Michel Foucault, Discipline and Punish: The Birth of Prison, New York: Random House, 1979. 
da cultura material, que é a sua fonte essencial, dizem respeito a todas as camadas de uma sociedade, seja ela qual for. Este artigo foi, portanto, concebido de uma perspectiva de democratização do saber, que leva em consideração a vida social e as identidades dos indivíduos comuns, geralmente invisíveis nas fontes escritas ou orais.

As fontes arqueológicas, uma vez que são materiais e locais, não oferecem simplesmente uma perspectiva deliberadamente local sobre os processos globais, mas, igual e fundamentalmente, permitem desnudar os silêncios na história. ${ }^{43}$ Têm assim um potencial único para recuar no tempo e comparar as mudanças dos períodos pré-coloniais, coloniais e pós-coloniais. Essa perspectiva de longa duração é importante no sentido de que permite colocar em relevo os diferentes estratos da evolução da vida social, econômica e política e, daí, determinar os atores, as causas e as consequências. Foi isso que tentamos fazer neste artigo.

\title{
Texto recebido em 23/11/2010 e aprovado em 20/3/2011
}

\begin{abstract}
Resumo
Neste artigo, confrontam-se dados arqueológicos e materiais com fontes clássicas da história para esclarecer as trajetórias históricas, sobre a gestão e a ocupação do espaço e reivindicações identitárias na Senegâmbia. Para tal é realizada breve avaliação dos saberes e dos limites da produção arqueológica, histórica, antropológica e etnográfica dessa região, que se configura como um mosaico de espaços e populações, marcadas por conflitos, negociações e acordos ao longo dos anos, constituindo-se como uma região culturalmente plural. A arqueologia histórica pode contribuir para os debates relacionados à incorporação das sociedades senegambianas nas correntes de mudanças do sistema capitalista e no seu impacto sobre a vida cotidiana e para avaliar a importância, a natureza e o papel dos produtos das trocas nas economias locais, em razão do caráter fragmentário das fontes escritas a esse respeito.
\end{abstract}

Palavras chave: Senegâmbia - identidades - arqueologia histórica.

43 Ann Brower Stahl, Making History in Banda. Anthropological Visions of Africa's Past, Cambridge: Cambridge University Press, 2001. 


\begin{abstract}
This article compares archeological data and materials with classic historical sources in examining the relationship of historical trajectories of the management and occupation of space to issues of ethnic identity in Senegambia. To this end, there is a brief analysis of the production of archeological, historical, anthropological and ethnographic knowledges about this region, as well as of the limits of these bodies of knowledge, which are configured like a mosaic of spaces and populations that are marked over the years by conflicts, negotiations and accords, thus constituting a culturally diverse region. Historical archeology can contribute to discussions related to the incorporation of Senegambian societies in the currents of changes to the capitalist system and its impact on everyday life. It can also assist in evaluating the importance, nature and role of the products that formed the basis of trade in local economies, given the fragmentary nature of written sources on this subject.
\end{abstract}

Keywords: Senegambia - identities - historical archeology 